\title{
Convergent finite elements for a class of nonconvex variational problems
}

\author{
BERNHARD KAWOHL $\dagger$ \\ Mathematisches Institut, Universität Köln, D-50923 Köln, Germany \\ AND \\ CHRISTOPH SCHWAB \\ Seminar für Angewandte Mathematik, ETH Zürich, Rämistrasse 101, \\ CH 8092 Zürich, Switzerland
}

[Received 24 November 1996 and in revised form 9 April 1997]

\begin{abstract}
We study the finite element discretization of the abstract minimization problem $\min \{F(u)\}$. The functional $F$ is neither convex nor growing at $\infty$. For the admissible class $C_{M}=\{u$ : $\Omega \rightarrow \mathbb{R}, u$ concave, $0 \leqslant u(x) \leqslant M$ \} polygonal domains $\Omega \subset \mathbb{R}^{2}$ and linear Courant triangles, we show the convergence of the finite element approximations to a minimizer of $F(u)$. A class of projected Newton methods for the discrete problems yields locally superlinear convergence. We present numerical experiments for a model functional $F$, related to Newton's problem of minimal resistance of a body moving through a fluid.
\end{abstract}

\section{Introduction}

One of the oldest problems in the Calculus of Variations is to determine a body of minimal resistance with respect to motion in a fluid. Isaac Newton gave a mathematical model for such motion in his Principia Mathematica (Newton (1686)). He imagined a fluid to consist of many particles with large mean free paths which interact only with the body under consideration through at most one perfectly elastic collision. Other effects, such as friction, turbulence, etc, are neglected in Newton's model. Until recently, Newton's model had been thoroughly studied only in the case of rotational bodies. For a discussion of Newton's simplifying assumptions as well as a survey of results in the rotational setting we refer for instance to Miele (1965).

In the paper by Buttazzo et al (1995), the authors studied existence questions for the nonradial case. If the body is described by

$$
\{(x, y) \mid \quad x \in \Omega, \quad 0 \leqslant y \leqslant u(x)\},
$$

then its resistance according to Newton is

$$
R(u)=\int_{\Omega} \frac{1}{1+|\nabla u|^{2}} \mathrm{~d} x .
$$

To minimize $R$, one has to choose a suitable class of admissible functions. Otherwise the infimum of $R$ is zero; it is approached through highly oscillatory arguments $u$.

Four different classes of admissible functions have been suggested in Buttazzo et al

† Email: kawohl@mi.uni-koeln.de 
(1995). For the reader's convenience, let us recall two of them for which the infimum of $R$ is indeed attained. The first class is

$$
C_{M}=\left\{u: \Omega \rightarrow \mathbb{R} \mid 0 \leqslant u \leqslant M, \Omega \subset \mathbb{R}^{2}, u \text { concave }\right\} .
$$

Notice that bounded concave functions are locally Lipschitz continuous so that $R$ is welldefined on $C_{M}$. The second class is

$$
E_{M}=\left\{u \in H_{\mathrm{loc}}^{1}(\Omega) \mid 0 \leqslant u \leqslant M, \Delta u \leqslant 0\right\}
$$

where $\Delta u \leqslant 0$ has to be understood in the distributional sense, i.e.

$$
\int_{\Omega} \nabla u \cdot \nabla \varphi \mathrm{d} x \geqslant 0 \quad \text { for all } \varphi \in C_{0}^{\infty}(\Omega), \quad \varphi \geqslant 0 .
$$

The resistance is well-defined for functions $u \in E_{M}$, too. A class of functionals more general than $R$ was studied in Buttazzo et al (1995), namely

$$
F(u)=\int_{\Omega} f(x, u, \nabla u) \mathrm{d} x
$$

where

$$
f(x, u, p) \text { is lower semi-continuous on } \mathbb{R} \times \mathbb{R}^{n} \text { for a.e. } x \in \Omega \subset \mathbb{R}^{n} \text {. }
$$

We summarize the main results of Buttazzo et al (1995) in

THEOREM 1.1 Assume that (1.5) holds. Then, for every $M>0$, there exists a solution to the problem

$$
\min \left\{F(u): u \in C_{M}\right\}
$$

Note that the function $f(x, u, p)=\left(1+|p|^{2}\right)^{-1}$ in Newton's problem is smooth and bounded. The key ingredient in the existence proof for (1.6) is the following compactness property of $C_{M}$ (see, e.g., Rockafellar (1970), Lions (1981), Buttazzo et al (1995)) which we will also use below.

PROPOSITION 1.1 For every $M>0$ and every $1 \leqslant p<\infty$, the class $C_{M}$ is compact with respect to the strong topology of $W_{\text {loc }}^{1, p}(\Omega)$.

The class $E_{M}$ owes its name to an analogous entropy constraint for highly nonunique transonic flow problems (Feistauer \& Nečas (1985)) where physically relevant solutions are located in $E_{M}$. We emphasize that the functional $F$ is in general neither convex nor coercive and that the Euler equation formally associated with $F$ is of mixed elliptic-hyperbolic type. Thus, smoothness or uniqueness of minimizers can generally not be expected.

The purpose of the present paper is the analysis of convergent finite element approximations to solutions of (1.6). It is organized as follows. In Section 2 we present a finite element discretization of the problem (1.6) and prove its convergence. This finite element discretization is nonconforming in the sense that the nonlocal concavity constraint is relaxed and replaced by a suitable penalty functional. We prove that as the penalty parameter $\varepsilon^{-1}$ increases (at fixed $N$ ), the approximate solutions $u_{\varepsilon}^{N}$ converge to concave functions $u^{N}$ 
that are conforming approximations of (1.6). As a byproduct we prove that these conforming approximations $u^{N}$ converge also to a minimizer of (1.6).

We make the assumption that the finite dimensional nonlinear problems resulting from the discretization process are solved exactly. In Section 3 we address the numerical solution of the finite dimensional nonlinear problems. A class of descent algorithms with line search due to Bertsekas (1982) is presented and sufficient conditions for their convergence are obtained, in terms of the data given in the variational problem. In Section 4, we present numerical results for $\Omega=(-1,1)^{2}$ which strongly indicate that even interior regularity, i.e. regularity away from $\partial \Omega$ and the 'active set'

$$
\mathcal{A}=\{x \in \Omega \mid u(x)=0 \text { or } u(x)=M\},
$$

does not hold for the minimizers $u$ of (1.6) in polygonal domains $\Omega$. An important feature of the finite dimensional minimization algorithm is that the finite dimensional approximation of the active set $\mathcal{A}$ is identified in finitely many steps and, once this has happened, the rate of convergence is locally superlinear, provided the density $f(x, u, p)$ in (1.4) is sufficiently regular.

Throughout the paper we denote by $c$ a generic constant and assume that

$$
\Omega \subset \mathbb{R}^{2} \text { is a polygonal domain, } f(x, u, p)=f(p): \mathbb{R}^{2} \rightarrow[0, \infty) .
$$

\section{Finite element discretization}

We discretize (1.6) using piecewise linear Courant finite elements. To this end, let $\left\{\mathcal{T}_{N}\right\}$ be a regular family of quasiuniform triangulations of $\Omega$ of meshwidth $h(N) \leqslant 1$ which consist of $K(N)$ open triangles $T_{k}, k=1, \ldots, K(N)$. Here and in what follows, $N=O\left(h^{-2}\right)$ is the number of nodes in the triangulation $\mathcal{T}_{N}$ and regular means that $\bar{T}_{k} \cap \bar{T}_{\ell}$ is either empty, a node or an entire side $\bar{e}_{k \ell}$. The smallest angle of $T_{k} \in \mathcal{T}_{N}$ is positive, uniformly in $N$. We denote by $\mathcal{E}_{N}$ the set of all interelement edges and by $\mathcal{N}_{N}$ the set of nodes of the triangulation $\mathcal{T}_{N}$.

On $\mathcal{T}_{N}$, we introduce the space of continuous, piecewise linear functions

$$
S^{N}=\left\{u\left|u \in C^{0}(\bar{\Omega}), \quad u\right|_{T_{k}} \text { is linear } \forall T_{k} \in \mathcal{T}_{N}\right\}
$$

Obviously, every $u \in S^{N}$ is uniquely determined by its values in the nodes $P \in \mathcal{N}_{N}$.

The assumptions (1.8) ensure that the value of the functional $F$ in (1.4) can be determined for piecewise linear functions $u \in S^{N}$ without numerical integration and other variational crimes, since

$$
F(u)=\sum_{T_{k} \in \mathcal{T}_{N}}\left|T_{k}\right| f\left(\left.\nabla u\right|_{T_{k}}\right), \quad u \in S^{N}
$$

as $\nabla u$ is constant on $T_{k} \in \mathcal{T}_{N}$ if $u \in S^{N}$.

Since we wish to approximate minimizers of (1.4) in the set $C_{M}$, it will be necessary to restrict $S^{N}$ further to

$$
S_{M}^{N}:=S^{N} \cap\{u \mid 0 \leqslant u \leqslant M, \quad x \in \Omega\} .
$$


Evidently, $u \in S_{M}^{N}$ if and only if $0 \leqslant u(P) \leqslant M$ for all $P \in \mathcal{N}_{N}$. The concavity constraint will not be incorporated into $S_{M}^{N}$ directly, but will be enforced via a suitable penalty functional which we now describe.

We begin with the observation that $u \in S^{N}$ as a mapping from $\Omega$ into $\mathbb{R}$ is concave if and only if $-\nabla u$ is a monotone operator or, equivalently, if and only if

$$
\left(\left.\nabla u\right|_{T_{k}}-\left.\nabla u\right|_{T_{\ell}}\right) \cdot \vec{n}_{k \ell} \geqslant 0 \quad \text { for every } k, \ell .
$$

Here $T_{k}$ and $T_{\ell}$ are adjacent triangles sharing a common edge $e_{k \ell}$ and $\vec{n}_{k \ell}$ is the unit normal on $e_{k \ell}$ pointing from $T_{k}$ into $T_{\ell}$. Alternatively, concave functions $u(x)$ satisfy the inequality

$$
u(y) \leqslant u(x)+\nabla u(x) \cdot(y-x) \text { a.c. } x, y \in \Omega .
$$

If (2.4) is violated, then $u$ is not concave. To penalize the nonconcavity of $u^{N}$ we define, for a parameter $1 \leqslant s<\infty$ at our disposal, the constraint functional

$$
\mathcal{C}^{N}(u):=\sum_{e_{k \ell} \in \mathcal{E}_{N}}\left|\left[\left(\left.\nabla u\right|_{T_{k}}-\left.\nabla u\right|_{T_{\ell}}\right) \cdot \vec{n}_{k \ell}\right]^{-}\right|^{s}, \quad u \in S^{N} .
$$

Notice that $\mathcal{C}^{N}$ depends on the triangulation $\mathcal{T}_{N}$. By (2.4) we have further that

$$
\mathcal{C}^{N}(v)=0 \quad \forall v \in S^{N} \cap C_{M} .
$$

Therefore we study the family of finite dimensional problems

$$
\min _{v \in S_{M}^{N}}\left\{F(v)+\frac{1}{\varepsilon} \mathcal{C}^{N}(v)\right\}
$$

where $\varepsilon=\varepsilon(N)$ tends to zero as $N \rightarrow \infty$. We call a family of minimizers

$$
u_{\varepsilon}^{N}=\arg \min _{v \in S_{M}^{N}}\left\{F(v)+\frac{1}{\varepsilon} \mathcal{C}^{N}(v)\right\}
$$

finite element solutions of the variational problem (1.6).

THEOREM 2.1 Let $1 \leqslant s<\infty$ be given.

(i) Assume that $f \in C^{k, \lambda}\left(\mathbb{R}^{2}\right)$, the set of continuous functions whose derivatives up to order $k$ are Hölder continuous with exponent $\lambda \in(0,1]$.

Then

$$
\begin{gathered}
\left.F(\cdot)\right|_{S_{M}^{N} \in C^{k, \lambda}\left([0, M]^{N}\right)} \\
\left.\mathcal{C}^{N}(\cdot)\right|_{S_{M}^{N} \in C^{\lfloor s\rfloor, s-\lfloor s\rfloor}\left([0, M]^{N}\right)}
\end{gathered}
$$

where the functionals are interpreted as functions of the nodal variables $u^{N}\left(P_{i}\right)$ of $u^{N} \in S_{M}^{N}$ and $\lfloor s\rfloor:=\max \{x \in \mathbb{Z} \mid x<s\}$.

(ii) Assume (1.5), (1.8) and that $f \in C_{b}^{0}\left(\mathbb{R}^{2}\right)$, the set of continuous and bounded functions on $\mathbb{R}^{2}$. Assume further that $\Omega$ is convex. Then for every $\varepsilon>0$ and every $N$ there exist finite element solutions $u_{\varepsilon}^{N}$. Moreover, they are almost concave in the sense that they satisfy the $a$ priori estimate

$$
u_{\varepsilon}^{N}(y) \leqslant u_{\varepsilon}^{N}(x)+\nabla u_{\varepsilon}^{N}(x) \cdot(y-x)+c \varepsilon^{1 / s} h^{(1-s) / s}|x-y|
$$


for every $x, y \in \Omega$ where the constant $C$ depends only on the density $f$, the domain $\Omega$ and the family $\left\{\mathcal{T}_{N}\right\}$ of triangulations but is independent of $\varepsilon, x, y$ and $N$.

(iii) Select in (2.8) a sequence $\{\varepsilon(N)\}_{N}$ of penalty parameters satisfying

$$
\varepsilon(N) \leqslant\left\{\begin{array}{lc}
c(h(N))^{1 / \alpha} & 0<\alpha<1 /(s-1), s \in(1, \infty), \\
o(1) & s=1 .
\end{array}\right.
$$

Under the assumptions (i) and (ii), the corresponding sequence $\left\{u_{\varepsilon(N)}^{N}\right\}$ of finite element solutions obtained from (2.8) contains a subsequence $\left\{u_{\varepsilon\left(N^{\prime}\right)}^{N^{\prime}}\right\}$ which converges to a limit $u \in C_{M}$ in $W_{\text {loc }}^{1, p}(\Omega), 1 \leqslant p<\infty$. Moreover, the limit $u$ of the subsequence of finite element solutions solves (1.6).

In the proof of Theorem 2.1, we use the following density result.

LEMMA 2.1 For every $u \in C_{M}$ there exists a sequence $\left\{u_{N}\right\}_{N} \subset C_{M} \cap S^{N}$ such that $u_{N} \rightarrow u$ in $W_{\mathrm{loc}}^{1, p}(\Omega)$ for $1 \leqslant p<\infty$.

Proof. For $u \in C_{M}$ and every $\tilde{\Omega} \subset \subset \Omega$, we have from (2.5) for $x \in \tilde{\Omega}$ and $y \in \partial \Omega$ such that $y-x$ and $\nabla u(x)$ point in the same direction

$$
|\nabla u(x)| \leqslant \frac{2 M}{\operatorname{dist}(\tilde{\Omega}, \partial \Omega)} \quad \forall x \in \tilde{\Omega} .
$$

Hence $u \in C_{M}$ implies in particular that $u \in W^{1, \infty}(\tilde{\Omega})$.

Now let $\Omega^{\prime} \subset \subset \Omega$. Then there exists $N_{0}\left(\Omega^{\prime}\right)$ and a polygon $\Omega^{\prime \prime}$ such that

$$
\Omega^{\prime} \subseteq \Omega^{\prime \prime} \subset \subset \Omega, \quad \overline{\Omega^{\prime \prime}}=\bigcup_{T \in \mathcal{T}_{N}^{\prime \prime}} \bar{T} \quad \forall N \geqslant N_{0}
$$

for some subtriangulations $\mathcal{T}_{N}^{\prime \prime}$ of $\mathcal{T}_{N}$.

Let $u_{N}:=I_{N} u$ be the piecewise linear interpolant of $u \in C_{M}$. Then $u_{N} \in S^{N} \cap C_{M}$ and it follows from Theorem 3.1.4 of Ciarlet (1978) that

$$
\left\|u-u_{N}\right\|_{L^{\infty}\left(\Omega^{\prime \prime}\right)} \leqslant c h\|u\|_{W^{1, \infty}\left(\Omega^{\prime \prime}\right)} \rightarrow 0 \quad \text { as } \quad N \rightarrow \infty .
$$

Since $\Omega^{\prime} \subset \subset \Omega$ was arbitrary, we get (passing to a subsequence if necessary) that $u_{N} \rightarrow u$ in $C(\Omega)$. We claim that in fact $u_{N} \rightarrow u$ in $W^{1, p}\left(\Omega^{\prime \prime}\right), 1 \leqslant p<\infty$.

By the compactness result Proposition 1.1, the sequence $\left\{u_{N}\right\} \subset C_{M}$ has a subsequence $\left\{u_{N^{\prime}}\right\}$ which is Cauchy in $W^{1, p}\left(\Omega^{\prime \prime}\right)$ for $1 \leqslant p<\infty$ and any $\Omega^{\prime \prime} \subset \subset \Omega$ so that there exists a function $v \in C_{M}$ such that

$$
\left\|v-u_{N}\right\|_{W^{1, p}\left(\Omega^{\prime \prime}\right)} \rightarrow 0, \quad 1 \leqslant p<\infty, \quad \forall \Omega^{\prime \prime} \subset \subset \Omega .
$$

We claim that $v=u$ on $\Omega^{\prime \prime}$. To see this, select $2<p<\infty$ and observe that by the embedding $W^{1, p}\left(\Omega^{\prime \prime}\right) \subset L^{\infty}\left(\Omega^{\prime \prime}\right)$ (see, e.g., Theorem 5.4 of Adams (1978))

$$
\|u-v\|_{L^{\infty}\left(\Omega^{\prime \prime}\right)} \leqslant\left\|u-u_{N}\right\|_{L^{\infty}\left(\Omega^{\prime \prime}\right)}+c\left\|v-u_{N}\right\|_{W^{1, p}\left(\Omega^{\prime \prime}\right)} .
$$

Sending now $N \rightarrow \infty$ yields $v=u$ a.c. in $\Omega^{\prime \prime}$. Since $\Omega^{\prime} \subseteq \Omega^{\prime \prime} \subset \subset \Omega$ and $\Omega^{\prime}$ was arbitrary, the assertion follows. 
We can now turn to the proof of Theorem 2.1.

Proof of Theorem 2.1. To prove statement (i), we consider $F(\cdot)$ and $\mathcal{C}^{N}(\cdot)$ separately. For the smoothness of $F(\cdot)$, we use (2.2). Let $\vec{u}_{k} \in[0, M]^{3}$ denote the vector of nodal variables of $u \in S^{N}$ on $T_{k}$. Then $\left.\nabla u\right|_{T_{k}}=A_{k} \vec{u}_{k}$ where $A_{k} \in \mathbb{R}^{2 \times 3}$ is a certain matrix depending only on the nodes of $T_{k}$. Hence, by (1.8), $f\left(\left.\nabla u\right|_{T_{k}}\right)=f\left(A_{k} \vec{u}_{k}\right) \in C^{k, \lambda}\left([0, M]^{3}\right)$. Now, according to (2.2), $F(u)$ is a finite linear combination of such terms and therefore $F(.) \in$ $C^{k, \lambda}\left([0, M]^{N}\right)$.

Consider now $\mathcal{C}^{N}(\cdot)$. Again it suffices to verify that each term in the finite sum (2.6) is, as a function of the nodal values $\vec{u}_{k}$ and $\vec{u}_{\ell}$, in $C^{\lfloor s\rfloor, s-\lfloor s\rfloor}\left([0, M]^{4}\right)$. Since two of the six nodal values are identical due to the continuity of $u$ across $e_{k \ell}$, we denote the vector of the four 'essential' nodal values of $u$ on $T_{k} \cup T_{\ell}$ by $\vec{u}_{k \ell}$. Then a typical term in the sum (2.6) is $\left|\left[B_{k \ell} \vec{u}_{k \ell}\right]^{-}\right|^{s}$ where $B_{k \ell}$ is a certain matrix depending only on the node positions of $T_{k}$ and $T_{\ell}$. For this term, however, the smoothness is immediate.

To prove assertion (ii), we first show that $u_{\varepsilon}^{N}$ exists. To this end observe that by (i) $F(v)$ and $\mathcal{C}^{N}(v)$ are continuous functions of the nodal values $v\left(P_{i}\right)$ of $v \in S_{M}^{N}$ on the closed and bounded set $[0, M]^{N}$. Hence, for every $\varepsilon>0$ and every $N$, there exists a minimizer $u_{\varepsilon}^{N}$ of $F+\varepsilon^{-1} \mathcal{C}^{N}$ on $S_{M}^{N}$ by the Weierstrass Theorem.

To show (2.10), for a given triangulation $\mathcal{T}_{N}$ choose $x, y \in \bigcup_{i=1}^{K(N)} T_{i}$ and connect them by a straight line $\overline{x y} \subset \Omega$ (here the convexity of $\Omega$ is used). The assertion is only nontrivial if $\overline{x y}$ is not contained in one element $T_{j}$ which we assume from now on.

As a function of the arclength $t$ along $\overline{x y}, u_{\varepsilon}^{N}$ is a piecewise linear function $v(t)$ like the one depicted in Fig. 1 with breakpoints at the transversal intersections $t_{j}, j=1, \ldots, J-1$ of $\overline{x y}$ with certain interelement edges $\overline{e_{j-1, j}}$ across which $\nabla u_{\varepsilon}^{N}$ may jump.

The (weak) derivative $v^{\prime}(t)$ is piecewise constant, i.e.

$$
\left.v^{\prime}\right|_{\left(t_{j-1}, t_{j}\right)}=v_{j}^{\prime} \quad j=1, \ldots, J
$$

and

$$
\begin{aligned}
v\left(t_{J}\right) & =v_{J}^{\prime}\left(t_{J}-t_{J-1}\right)+v\left(t_{J-1}\right) \\
& =v_{J}^{\prime}\left(t_{J}-t_{J-1}\right)+v_{J-1}^{\prime}\left(t_{J-1}-t_{J-2}\right)+v\left(t_{J-2}\right) \\
& \cdots \\
& =v\left(t_{0}\right)+\sum_{j=1}^{J} v_{j}^{\prime}\left(t_{j}-t_{j-1}\right) .
\end{aligned}
$$

Hence

$$
v\left(t_{J}\right)-v\left(t_{0}\right)=v_{1}^{\prime}\left(t_{J}-t_{0}\right)+\sum_{j=2}^{J}\left(v_{j}^{\prime}-v_{j-1}^{\prime}\right)\left(t_{J}-t_{j-1}\right) .
$$

This yields the inequality

$$
v\left(t_{J}\right)-v\left(t_{0}\right) \leqslant v_{1}^{\prime}\left(t_{J}-t_{0}\right)+\sum_{j=2}^{J}\left[v_{j-1}^{\prime}-v_{j}^{\prime}\right]^{-}\left(t_{J}-t_{j-1}\right) .
$$

The estimate (2.10) will be derived from (2.14). 


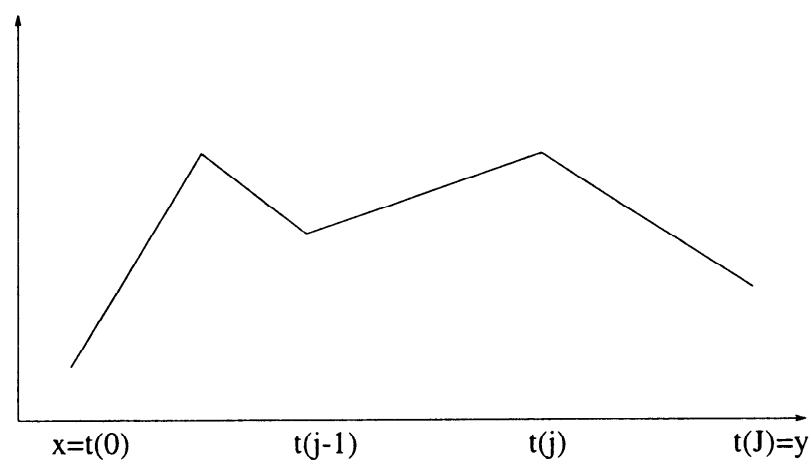

FIG. 1. The function $v(t)$ along the line $\overline{x y}$.

Assume first that $\overline{x y}$ does not intersect any node of $\mathcal{T}_{N}$. Then no interelement edge is completely contained in $\overline{x y}$ and each of the straight line segments in the intervals $\left(t_{j-1}, t_{j}\right)$ in Fig. 1 is contained in some triangle, $T_{j}$ say, of $\mathcal{T}_{N}$. As $u_{\varepsilon}^{N}$ is piecewise linear, $\nabla u_{\varepsilon}^{N}$ is constant on $T_{j}$ and we denote its value by $\nabla u_{\varepsilon}^{N}(j), j=1, \ldots, J$. Moreover, $v_{j}^{\prime}=$ $\vec{e}_{x y} \cdot \nabla u_{\varepsilon}^{N}(j)$ where $\vec{e}_{x y}=(y-x) /|y-x|$. Denoting $x_{0}=x, x_{J}=y$ and $x_{j}=\overline{x y} \cap \overline{e_{j-1, j}}$, $j=1, \ldots, J-1$, we get from (2.14) that

$$
\begin{aligned}
u_{\varepsilon}^{N}(y)-u_{\varepsilon}^{N}(x) \leqslant & \nabla u_{\varepsilon}^{N}(x) \cdot(y-x) \\
& +\sum_{j=2}^{J}\left[\left(\nabla u_{\varepsilon}^{N}(j-1)-\nabla u_{\varepsilon}^{N}(j)\right) \cdot \vec{e}_{x y}\right]^{-}\left|y-x_{j-1}\right| .
\end{aligned}
$$

Since the jump of $\nabla u_{\varepsilon}^{N}$ along the interelement edge $e_{j-1, j}$ is zero, an oblique jump can be estimated by the jump of $\nabla u_{\varepsilon}^{N}$ in the direction of the exterior unit normal $\vec{n}_{j-1, j}$ to $T_{j-1}$ pointing into $T_{j}$. This yields the bound

$$
\begin{aligned}
u_{\varepsilon}^{N}(y)- & u_{\varepsilon}^{N}(x) \leqslant \nabla u_{\varepsilon}^{N}(x) \cdot(y-x) \\
& +\sum_{j=2}^{J}\left[\left(\nabla u_{\varepsilon}^{N}(j-1)-\nabla u_{\varepsilon}^{N}(j)\right) \cdot \vec{n}_{j-1, j}\right]^{-}\left|y-x_{j-1}\right| \\
\leqslant & \nabla u_{\varepsilon}^{N}(x) \cdot(y-x) \\
& +\left(\sum_{j=2}^{J}\left|\left[\left(\nabla u_{\varepsilon}^{N}(j-1)-\nabla u_{\varepsilon}^{N}(j)\right) \cdot \vec{n}_{j-1, j}\right]^{-}\right|^{s}\right)^{1 / s}\left(\sum_{j=2}^{J}\left|y-x_{j-1}\right|^{s^{*}}\right)^{1 / s^{*}}
\end{aligned}
$$

by Hölder's inequality in $\mathbb{R}^{J-1}$ with $s^{*}=s /(s-1)$.

Since the triangulations $\mathcal{T}_{N}$ are quasiuniform, there exists a constant $c$ independent of $N$ such that $J \leqslant c|x-y| / h$. Therefore

$$
\sum_{j=2}^{J}\left|y-x_{j-1}\right|^{s^{*}} \leqslant J|y-x|^{s^{*}} \leqslant C(\operatorname{diam} \Omega)|x-y|^{s^{*}} / h .
$$


Inserting this into $(2.15)$ results in

$$
\begin{aligned}
u_{\varepsilon}^{N}(y)-u_{\varepsilon}^{N}(x) \leqslant & \nabla u_{\varepsilon}^{N}(x) \cdot(y-x) \\
& +c(\operatorname{diam} \Omega)^{1 / s^{*}} h^{-1 / s^{*}}\left(\mathcal{C}^{N}\left(u_{\varepsilon}^{N}\right)\right)^{1 / s}|x-y|
\end{aligned}
$$

with a constant $c$ independent of $\varepsilon$ and $N$.

Now $f(p) \geqslant 0$ and $u=M \in C_{M}$ imply

$$
0 \leqslant F\left(u_{\varepsilon}^{N}\right)+\frac{1}{\varepsilon} \mathcal{C}^{N}\left(u_{\varepsilon}^{N}\right) \leqslant F(M)=f(0)|\Omega|
$$

from where we get

$$
0 \leqslant \mathcal{C}^{N}\left(u_{\varepsilon}^{N}\right) \leqslant f(0)|\Omega| \varepsilon .
$$

Inserting this into (2.16), we obtain

$$
u_{\varepsilon}^{N}(y)-u_{\varepsilon}^{N}(x) \leqslant \nabla u_{\varepsilon}^{N}(x) \cdot(y-x)+c(\operatorname{diam} \Omega)^{1 / s^{*}}(f(0)|\Omega| \varepsilon)^{1 / s}|x-y| h^{-1 / s^{*}}
$$

which is (2.10). In the case that $\overline{x y}$ contains a node $P_{i}$ or an entire edge, we argue by continuity: (2.10) has been proved for almost all $x, y \in \Omega$ and $u_{\varepsilon}^{N}$ is continuous, therefore (2.10) is true everywhere.

To prove (iii), we note that (2.10) and (2.11) imply

$$
u_{\varepsilon(N)}^{N}(y)-u_{\varepsilon(N)}^{N}(x)-\nabla u_{\varepsilon(N)}^{N}(x) \cdot(y-x) \leqslant c(\varepsilon(N))^{\beta}|x-y|, \quad x, y \in \Omega
$$

where $\beta=(1-\alpha(s-1)) / s>0$. Functions in $C_{M}$ satisfy (2.18) with $c=0$ and exhibit, by Proposition 1.1, good compactness properties which allow us to apply the Direct Method in the Calculus of Variations (see, e.g., Dacorogna (1989)) to (1.6).

We shall extend this to functions satisfying (2.18). We proceed in several steps.

Step 1: We prove that $\left\{u_{\varepsilon(N)}^{N}\right\}_{N}$ contains a subsequence which converges in $W_{\text {loc }}^{1, p}(\Omega), 1 \leqslant$ $p<\infty$, to a limit $u \in C_{M}$.

For a given $x \in \Omega$ choose in (2.18) the point $y \in \partial \Omega$ in such a way that the vector $y-x$ points in the direction $-\nabla u_{\varepsilon(N)}^{N}(x)$ (i.e. into a direction of steepest descent of $\left.u_{\varepsilon(N)}^{N}\right)$. Then

$$
\left|\nabla u_{\varepsilon(N)}^{N}(x)\right| \leqslant \frac{u_{\varepsilon(N)}^{N}(x)-u_{\varepsilon(N)}^{N}(y)}{|x-y|}+c(\varepsilon(N))^{\beta} \leqslant \frac{M}{\operatorname{dist}(x, \partial \Omega)}+c(\varepsilon(N))^{\beta} .
$$

Therefore the sequence $\left\{u_{\varepsilon(N)}^{N}\right\}_{N}$ is uniformly bounded in $W_{\text {loc }}^{1, \infty}(\Omega)$.

Since $\left\{u_{\varepsilon(N)}^{N}\right\}_{N}$ is uniformly Lipschitz continuous on every $\Omega^{\prime} \subset \subset \Omega$, there exists a subsequence (again denoted $\left\{u_{\varepsilon(N)}^{N}\right\}_{N}$ ) which converges weakly in every $W^{1, p}\left(\Omega^{\prime}\right), 1 \leqslant$ $p<\infty$ and uniformly on $\Omega^{\prime}$ to a limit $u \in W_{\text {loc }}^{1, p}(\Omega), 1 \leqslant p<\infty$.

Let us show that $\nabla u_{\varepsilon(N)}^{N}$ converges pointwise almost everywhere in $\Omega^{\prime}$ to $\nabla u$. To this end, we adapt an idea of Marcellini (1990) (see also Rockafellar (1970)). Let $\mathbf{e}_{i}$ denote the unit vector in the direction of the $i$ th coordinate. Each $u_{\varepsilon(N)}^{N}$ is differentiable a.e. in $\Omega^{\prime}$ and so is $u$. Let $x$ be a point in which each $u_{\varepsilon(N)}^{N}$ and also $u$ are differentiable (the set of points 
where this is not the case is a countable union of measure zero sets and hence has measure zero). According to (2.10) we have for sufficiently small $\delta>0$ and any $\varepsilon>0$

$$
\begin{aligned}
u_{\varepsilon}^{N}\left(x+\delta \mathbf{e}_{i}\right)-u_{\varepsilon}^{N}(x) & \leqslant \delta D_{i} u_{\varepsilon}^{N}(x)+c \varepsilon^{\beta} \delta \\
& \leqslant u_{\varepsilon}^{N}(x)-u_{\varepsilon}^{N}\left(x-\delta \mathbf{e}_{i}\right)+2 c \varepsilon^{\beta} \delta .
\end{aligned}
$$

We select $\varepsilon=\varepsilon(N)$, send $N$ to infinity and obtain, upon dividing by $\delta>0$,

$$
\begin{gathered}
\quad \frac{u\left(x+\delta \mathbf{e}_{i}\right)-u(x)}{\delta}-c \lim _{N \rightarrow \infty}(\varepsilon(N))^{\beta} \leqslant \liminf _{N \rightarrow \infty} D_{i} u_{\varepsilon(N)}^{N} \\
\leqslant \limsup _{N \rightarrow \infty} D_{i} u_{\varepsilon(N)}^{N} \leqslant \frac{u(x)-u\left(x-\delta \mathbf{e}_{i}\right)}{\delta}+c \lim _{N \rightarrow \infty}(\varepsilon(N))^{\beta} .
\end{gathered}
$$

Next we send $\delta$ to zero and note that the upper and lower bounds for $D_{i} u_{\varepsilon(N)}^{N}$ converge to $D_{i} u$. Therefore

$$
\lim _{N \rightarrow \infty} \nabla u_{\varepsilon(N)}^{N}(x)=\nabla u(x) \text { a.c. } \quad x \in \Omega^{\prime} .
$$

By the Lebesgue dominated convergence theorem, the sequence $\left\{u_{\varepsilon(N)}^{N}\right\}$ converges to $u$ strongly in $W^{1, p}\left(\Omega^{\prime}\right), 1 \leqslant p<\infty$, for any $\Omega^{\prime} \subset \subset \Omega$. Since $\Omega^{\prime}$ was an arbitrary subset of $\Omega, \nabla u_{\varepsilon(N)}^{N} \rightarrow \nabla u$ pointwise a.e. in $\Omega$. Therefore we may also use the Lebesgue dominated convergence theorem to conclude that

$$
F\left(u_{\varepsilon(N)}^{N}\right) \rightarrow F(u)
$$

holds.

We claim that $u \in C_{M}$. To prove it, we observe that by (2.18) the limit $u(x)$ must satisfy (2.5). This implies that $u \in C_{M}$.

Step 2: Let $u^{N}$ be a minimizer of $F$ over $C_{M} \cap S^{N}$. We show that (a subsequence of) $\left\{u^{N}\right\}_{N}$ converges to a solution $\tilde{u}$ of (1.6).

We first show that for every $N$ a minimizer $u^{N}$ of $F$ over $C_{M} \cap S^{N}$ exists. To this end, we fix $\mathcal{T}_{N}$ and let $\varepsilon$ tend to zero. Since the sequence $\left\{u_{\varepsilon}^{N}\right\}_{\varepsilon>0}$ is determined by the nodal values $\left(u_{\varepsilon}^{N}\left(P_{1}\right), \ldots, u_{\varepsilon}^{N}\left(P_{N}\right)\right) \in[0, M]^{N}$ on the fixed triangulation $\mathcal{T}_{N}$, the theorem of Heine and Borel ensures that a subsequence $\left\{\left(u_{\varepsilon^{\prime}}^{N}\left(P_{1}\right), \ldots, u_{\varepsilon^{\prime}}^{N}\left(P_{N}\right)\right)\right\}_{\varepsilon^{\prime}>0}$ converges to some $\left(a_{1}, \ldots, a_{N}\right) \in[0, M]^{N}$. Denote by $u^{N} \in S_{M}^{N}$ the piecewise linear function with nodal values $u^{N}\left(P_{i}\right)=a_{i}$. Then, by construction,

$$
\left\|u_{\varepsilon^{\prime}}^{N}-u^{N}\right\|_{W^{1, \infty}(\Omega)} \rightarrow 0 \quad \text { as } \quad \varepsilon^{\prime} \rightarrow 0 .
$$

Moreover, since both $u_{\varepsilon^{\prime}}^{N}$ and $u^{N}$ are piecewise linear with function values in $[0, M]$ there exists a constant $A(N, M)$, independent of $\varepsilon^{\prime}$, such that

$$
\left\|\nabla u_{\varepsilon^{\prime}}^{N}\right\|_{L^{\infty}(\Omega)},\left\|\nabla u^{N}\right\|_{L^{\infty}(\Omega)} \leqslant A(N, M)
$$

(with the derivatives taken in the sense of distributions). Due to (2.21) and the uniform continuity of $f(p)$ on $[0, A]^{2}$ it follows that

$$
F\left(u_{\varepsilon^{\prime}}^{N}\right) \rightarrow F\left(u^{N}\right) \quad \text { as } \quad \varepsilon^{\prime} \rightarrow 0 .
$$


Further, due to (2.17) we have $0 \leqslant \mathcal{C}^{N}\left(u_{\varepsilon^{\prime}}^{N}\right) \leqslant f(0)|\Omega| \varepsilon^{\prime}$ which implies that $\mathcal{C}^{N}\left(u^{N}\right)=0$. Therefore $u^{N} \in S^{N} \cap C_{M}$. Moreover, we claim that

$$
F\left(u^{N}\right)=\min _{v \in S^{N} \cap C_{M}} F(v) .
$$

To prove it we observe that the inclusion $S^{N} \cap C_{M} \subset S_{M}^{N}$ implies

$$
F\left(u_{\varepsilon^{\prime}}^{N}\right) \leqslant F\left(u_{\varepsilon^{\prime}}^{N}\right)+\frac{1}{\varepsilon^{\prime}} \mathcal{C}^{N}\left(u_{\varepsilon^{\prime}}^{N}\right) \leqslant F(v)+\frac{1}{\varepsilon^{\prime}} \mathcal{C}^{N}(v)=F(v)
$$

for every $\varepsilon^{\prime}>0$ and every $v \in S^{N} \cap C_{M}$ since $\mathcal{C}^{N}(v)=0$. We let $\varepsilon^{\prime}$ tend to zero and use (2.22) to obtain (2.23).

By Proposition 1.1, (a subsequence of) $\left\{u^{N}\right\}$ converges strongly in $W_{\text {loc }}^{1, p}(\Omega), 1 \leqslant p<$ $\infty$, to a limit $\tilde{u} \in C_{M}$.

We claim that $\tilde{u}$ is a minimizer of $F$ on $C_{M}$. To prove this, let $v \in C_{M}$ be given. Then, by Lemma 2.1, $v$ can be approximated by a sequence $v^{N} \in C_{M} \cap S^{N}$ in $W^{1, p}\left(\Omega^{\prime}\right)$ for every $\Omega^{\prime} \subset \subset \Omega, 1 \leqslant p<\infty$.

Now pick $\Omega^{\prime} \subset \subset \Omega$ such that the set $\Omega \backslash \Omega^{\prime}$ has measure less than $\varepsilon_{1}$, say. Then pick $N$ sufficiently large so that $\left\|v-v^{N}\right\|_{W^{1, p}\left(\Omega^{\prime}\right)} \leqslant \varepsilon_{2}$. Since the density $f(p)$ is uniformly bounded we have

$$
F(v) \geqslant \int_{\Omega^{\prime}} f(\nabla v) \mathrm{d} x-\varepsilon_{1}\|f\|_{L^{\infty}},
$$

and since $f$ is uniformly continuous on bounded sets, due to Egorov's theorem

$$
\int_{\Omega^{\prime}} f(\nabla v) \mathrm{d} x-O\left(\varepsilon_{1}\right) \geqslant \int_{\Omega^{\prime}} f\left(\nabla v^{N}\right) \mathrm{d} x-O\left(\varepsilon_{1}\right)-O\left(\varepsilon_{2}\right)
$$

with $O\left(\varepsilon_{2}\right)$ uniform in $N$. Combining this with (2.24) and using the minimizing property (2.23) of $u^{N}$ gives

$$
F(v) \geqslant F\left(v^{N}\right)-O\left(\varepsilon_{1}\right)-O\left(\varepsilon_{2}\right) \geqslant F\left(u^{N}\right)-O\left(\varepsilon_{1}\right)-O\left(\varepsilon_{2}\right) .
$$

By the same arguments, choosing $\Omega^{\prime}$ as above and $N$ such that $\left\|\tilde{u}-u^{N}\right\|_{W^{1, p}\left(\Omega^{\prime}\right)}<\varepsilon_{2}$, we obtain

$$
F\left(u^{N}\right) \geqslant F(\tilde{u})-O\left(\varepsilon_{1}\right)-O\left(\varepsilon_{2}\right)
$$

Now (2.25) and (2.26) lead to

$$
F(v) \geqslant F(\tilde{u})-O(\varepsilon) \quad \text { for any } v \in C_{M}, \varepsilon>0,
$$

that is $\tilde{u}$ minimizes $F$ on $C_{M}$.

Step 3: Finally we show that the limit $u$ of (a subsequence of) the sequence $u_{\varepsilon(N)}^{N}$ of the FE solutions obtained in Step 1 satisfies $F(u)=F(\tilde{u})$, i.e. $u$ is a minimizer of $F$ on $C_{M}$.

To this end, observe that for the sequence $\left\{u^{N}\right\}$ from Step 2 we have

$$
F\left(u^{N}\right)=F\left(u^{N}\right)+\frac{1}{\varepsilon(N)} \mathcal{C}^{N}\left(u^{N}\right) \geqslant F\left(u_{\varepsilon(N)}^{N}\right)+\frac{1}{\varepsilon(N)} \mathcal{C}^{N}\left(u_{\varepsilon(N)}^{N}\right) \geqslant F\left(u_{\varepsilon(N)}^{N}\right) .
$$


As shown in Step 2, after passing to a subsequence if necessary, the left-hand side converges to $F(\tilde{u})$.

By (2.20) and (2.17), the right-hand side converges to $F(u)$ and therefore we have shown $F(\tilde{u}) \geqslant F(u)$. Since $\tilde{u} \in C_{M}$ was a minimizer, so is $u$. This completes the proof.

REMARK 2.1 The boundedness of the density $f$ was used only in Step 2 of the above proof.

REMARK 2.2 If the minimizer $u$ is unique, the whole sequence $u_{\varepsilon(N)}^{N}$ converges to $u$.

REMARK 2.3 Step 2 of the proof shows in particular that conforming (i.e. concave) FEapproximations $u^{N} \in C_{M} \cap S^{N}$ exist and, possibly after passing to a subsequence, converge to a minimizer $u$.

\section{Minimization of the discretized problems}

In the previous sections we showed convergence results for sequences of finite element solutions $u_{\varepsilon}^{N}$ and $u^{N}$ of (1.6). These solutions, however, must be computed by solving a finite dimensional minimization problem of the form

$$
\min _{[0, M]^{N}} G(u)
$$

where $u=\left(u_{1}, \ldots, u_{N}\right) \in \mathbb{R}^{N}$ denotes now the vector of nodal values of the finite element solution and $G(\cdot)$ is the objective function corresponding to (2.7). Our purpose in the present section is to introduce a class of algorithms suitable for the finite dimensional minimization and to present corresponding convergence results from Bertsekas (1982) which are valid in our setting. Throughout, we assume that $G$ is at least in $C^{1,1}\left([0, M]^{N}\right)$, i.e. that

$$
|\nabla G(x)-\nabla G(y)| \leqslant L|x-y| \quad \forall x, y \in[0, M]^{N} .
$$

According to Theorem 2.1 this is the case if $s \geqslant 2$ and if $f(p) \in C^{1,1}\left(\mathbb{R}^{2}\right)$.

The discrete minimization (3.1) can be performed with a class of projected descent algorithms which we now describe. To this end, we denote

$$
[x]^{\#}:=\max (0, \min (x, M)), \quad x \in \mathbb{R},
$$

and we write for $u \in \mathbb{R}^{N}$

$$
[u]^{\#}:=\left(\left[u_{1}\right]^{\#},\left[u_{2}\right]^{\#}, \ldots,\left[u_{N}\right]^{\#}\right) .
$$

The first-order necessary conditions for a vector $\bar{u}$ to be a local minimum of (3.1) are

$$
\partial_{i} G(\bar{u}) \geqslant 0 \quad \text { if } \bar{u}_{i}=0, \quad \partial_{i} G(\bar{u}) \leqslant 0 \quad \text { if } \bar{u}_{i}=M, \quad \partial_{i} G(\bar{u})=0 \quad \text { else. }
$$

Here and in what follows, $\partial_{i}$ denotes the partial derivative with respect to the $i$ th coordinate.

The minimization algorithms we consider are of the form (Bertsekas (1982))

$$
u^{(k+1)}=u^{(k)}\left(\alpha_{k}\right), \quad u^{(k)}(\alpha)=\left[u^{(k)}+\alpha p^{(k)}\right]^{\#}, \quad \alpha \geqslant 0
$$


where $\alpha_{k}$ is a stepsize and $p^{(k)}$ is a descent direction, such as for example

$$
p^{(k)}=-D^{(k)} \nabla G\left(u^{(k)}\right) .
$$

Here $D^{(k)}$ is a sequence of positive definite, symmetric scaling matrices still at our disposal. According to Bertsekas (1982), the sequence of matrices $D^{(k)}$ and the stepsizes $\alpha_{k}$ will be chosen as follows. Let a small number $\delta>0$ and parameters $\beta \in(0,1), \sigma \in\left(0, \frac{1}{2}\right)$ be given. Define

$$
w_{k}=\left|u^{(k)}-\left[u^{(k)}-\nabla G\left(u^{(k)}\right)\right]^{\#}\right|, \quad \delta_{k}=\min \left(\delta, w_{k}\right) .
$$

Then the index set $I_{k}^{\#}$ of active constraints at iteration $k$ is

$$
I_{k}^{\#}=\left\{i \mid 0 \leqslant u_{i}^{(k)} \leqslant \delta_{k}, \partial_{i} G\left(u^{(k)}\right)>0 \text { or } M-\delta_{k} \leqslant u_{i}^{(k)} \leqslant M, \partial_{i} G\left(u^{(k)}\right)<0\right\} .
$$

The scaling matrices $D^{(k)}$ are assumed symmetric, positive definite and diagonal with respect to $I_{k}^{\#}$, i.e.

$$
D_{i j}^{(k)}=0 \quad \forall i, j \in I_{k}^{\#}, i \neq j .
$$

The stepsizes $\alpha_{k}$ in (3.4) are given by

$$
\alpha_{k}=\beta^{m_{k}}
$$

where $m_{k}$ is the first nonnegative integer such that

$$
G\left(u^{(k)}\right)-G\left(u^{(k)}\left(\beta^{m}\right)\right) \geqslant \sigma\left\{\beta^{m} \sum_{i \notin I_{k}^{*}} \partial_{i} G\left(u^{(k)}\right) p_{i}^{(k)}+\sum_{i \in I_{k}^{*}} \partial_{i} G\left(u^{(k)}\right)\left[u_{i}^{(k)}-u_{i}^{(k)}\left(\beta^{m}\right)\right]\right\} .
$$

For the minimization algorithm thus defined, we have the following convergence result (Bertsekas (1982)).

PROPOSITION 3.1 Assume that the matrices $D^{(k)}$ are diagonal with respect to the index sets $I_{k}^{\#}$ and uniformly positive definite, i.e. there exist $\lambda_{i}>0$ independent of $k$ such that

$$
\lambda_{1}|x|^{2} \leqslant x^{\top} D^{(k)} x \leqslant \lambda_{2}|x|^{2} \quad \forall x \in \mathbb{R}^{N}, \quad k=0,1, \ldots
$$

and that (3.2) holds. Then every limit point of a sequence $\left\{u^{(k)}\right\}$ generated by the iteration (3.4)-(3.8) is a critical point of (3.1).

REMARK 3.1 A basic special case is the method of steepest descent with projection which is obtained by selecting in (3.4) $\alpha_{k}=\bar{\alpha}>0$ sufficiently small and $D^{(k)}$ in (3.5) to be the unit matrix. This method converges linearly.

It turns out that under some additional assumptions on $G$ and the minimum $\bar{u}$ the algorithm (3.4), (3.5) displays local superlinear convergence. To describe these assumptions, define for $u \in[0, M]^{N}$ the set $B(u)$ of indices of binding constraints at $u$, i.e.

$$
B(u)=\left\{i \mid u_{i}=0 \text { or } u_{i}=M\right\} .
$$

Consider now an isolated local minimum $\bar{u}$ of $G$ for which there exists $\bar{\delta}>0$ such that 
$G \in C^{2}$ in the open ball of radius $\bar{\delta}$ with centre at $\bar{u}$ and there exist further $0<\lambda_{1} \leqslant \lambda_{2}$ such that for all $x \in \mathbb{R}^{N}$ with $x_{i}=0$ for $i \in B(\bar{u})$

$$
\lambda_{1}|x|^{2} \leqslant x^{\top} H[G(u)] x \leqslant \lambda_{2}|x|^{2} \quad \forall|u-\bar{u}|<\bar{\delta}
$$

where $H[G(u)]$ denotes the Hessian matrix of second derivatives of $G(\cdot)$ at $u$. In addition, we require (3.3). The following result from Bertsekas (1982) shows an important property of the descent algorithm, namely that the set $B(\bar{u})$ is identified after finitely many steps, i.e.

$$
I_{k}^{\#}=B\left(u^{(k)}\right)=B(\bar{u}) \quad \forall k \geqslant \bar{k} .
$$

Proposition 3.2 Let $\bar{u}$ be a local minimum of $G$ satisfying (3.10) and (3.3) and assume that

$$
0<\bar{\lambda}_{1} \leqslant D_{i i}^{(k)} \quad i \in I_{k}^{\#}, \quad k=0,1, \ldots .
$$

Then, if $u^{(0)}$ is sufficiently close to $\bar{u}$, the sequence $u^{(k)}$ generated by (3.4)-(3.8) converges to $\bar{u}$ and (3.11) holds.

This implies in particular that for $k \geqslant \bar{k}$ in (3.11) the descent algorithm with projection reduces to an unconstrained one operating on the free indices $\{1, \ldots, N\} \backslash B(\bar{u})$. By a suitable selection of the matrices $D^{(k)}$, e.g. portions of the inverse Hessian of $G$ at $u^{(k)}$ or quasiNewton update approximations of it, locally quadratic resp. superlinear convergence rates are achieveable under standard assumptions, such as $G \in C^{3}\left([0, M]^{N}\right)$. By Theorem 2.1, this holds for densities $f(p) \in C^{3}\left(\mathbb{R}^{2}\right)$ and $s>3$.

\section{Numerical results}

In the present section we report numerical results obtained from a finite element discretization of (1.6) with $F(u)=R(u)$ and the algorithm (3.4), (3.5). We solved (1.6) on the domain $\Omega=(-1,1)^{2}$ and reduced the finite element calculation to one quadrant $(0,1)^{2}$ using the symmetry conditions

$$
u\left(x_{1}, x_{2}\right)=u\left(x_{1},-x_{2}\right)=u\left(-x_{1}, x_{2}\right)=u\left(-x_{1},-x_{2}\right) .
$$

REMARK 4.1 Obviously the domain $(-1,1)^{2}$ and the functional are invariant under the variable changes (4.1). It is an open question whether this implies the corresponding symmetry for the minimizers, i.e. the existence of asymmetric minimizers for symmetric domains cannot be ruled out at this point.

All statements which follow in this section refer to the domain $(0,1)^{2}$.

We used a uniform mesh consisting of triangles obtained from a subdivision on $(0,1)^{2}$ into $N^{2}$ quadrilaterals and bisection of each quadrilateral along the diagonal parallel to $x_{1}=x_{2}$. We started the iterations from the initial, concave profile

$$
u\left(x_{1}, x_{2}\right)=M\left(1-\frac{x_{1}^{2}+x_{2}^{2}}{2}\right) .
$$

The sequence of scaling matrices $D^{(k)}$ in (3.5) was selected to be the unit matrix and, according to Remark 3.1, the stepsizes $\alpha_{k}$ were selected constant. The value of $\varepsilon$ which 


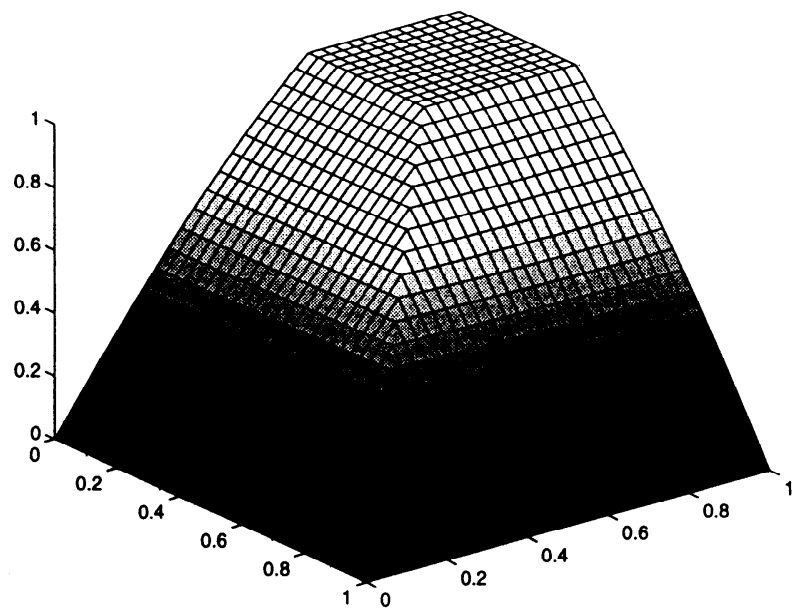

FIG. 2. Profile of $u_{\varepsilon}^{N}$ for $M=1$.

TABLE 1

Edge lengths $\ell$ of the discrete active sets $\mathcal{A}^{N}$ in dependence on $M$.

\begin{tabular}{cccc}
\hline$M$ & 1 & 2 & 3 \\
\hline$\ell$ & $3 / 8$ & $1 / 4$ & $1 / 8$
\end{tabular}

was important for the convergence of the scheme, was actually chosen fairly large, in our case we selected $\varepsilon=20$. This choice of $\varepsilon$ was sufficient to keep the value of $\mathcal{C}\left(u_{\varepsilon}^{N}\right)$ below 0.01 throughout the descent iterations. Selecting a much smaller value of $\varepsilon$ increases the stiffness of the discrete problem substantially and reduces the feasible stepsizes. We performed 10000 steps of (3.4) (with parameter $\delta=0.01$ ) which, due to the explicit nature of the algorithm, can be executed quite efficiently. In this fashion we obtained profiles $u_{\varepsilon}^{N}(x)$ for $M=1,2,3$ and a meshwidth of $h=1 / 32$ (corresponding to $N=1024$ degrees of freedom) corresponding to nodal values of $(i h, j h), 0 \leqslant i, j \leqslant 32$. Figures 2,3 and 4 depict the profiles thus obtained for $M=1,2,3$, respectively.

The approximate minimizers found in this fashion exhibit the following structure. They all have a discrete active set $\mathcal{A}^{N}$ which is a square centred at the origin and with the edge lengths listed in Table 1. It appears that the edge length is decreasing as $M$ increases, i.e. as the profile gets more slender. As is visible in Fig. 3, the profile is nondifferentiable at $\partial \mathcal{A}^{N}$. However, unlike the unsmoothness encountered for elliptic problems with constraints (as, for example, the obstacle problem), here the gradient of $u$ (rather than the second derivatives) has a jump at $\partial \mathcal{A}$. Moreover, the profiles exhibit a smoothly curved edge emanating from each vertex of $\mathcal{A}^{N}$ and connecting the vertex of $\mathcal{A}^{N}$ with the nearest 


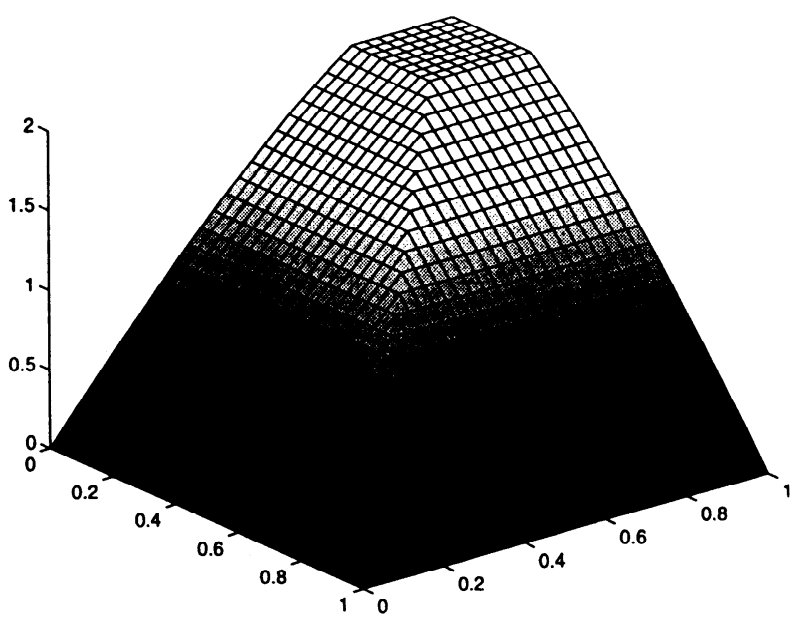

FIG. 3. Profile of $u_{\varepsilon}^{N}$ for $M=2$.

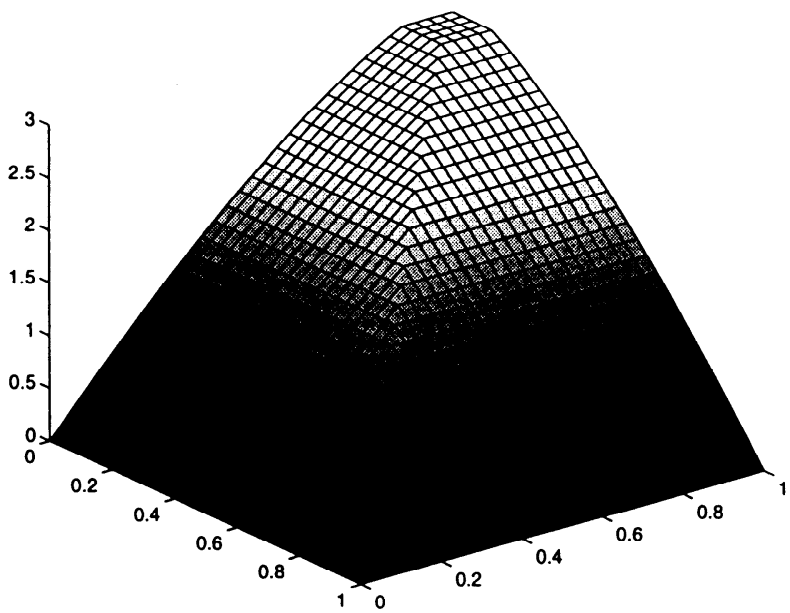

FIG. 4. Profile of $u_{\varepsilon}^{N}$ for $M=3$.

corner of $(-1,1)^{2}$. In Fig. 5 we sketch schematically the domains where $u_{\varepsilon}^{N}$ appears to be smooth. The set $\mathcal{A}^{N}$ corresponds to $I$. For $M=2$ and $M=3$, the profile in the subregions labelled II appears to be a developable surface parallel to the edge of $\mathcal{A}^{N}$. In Table 2, we show the nodal function values for $\left(u_{\varepsilon}^{N}\right)_{i j}$ for $M=2$ and $j=9$, i.e. along the first vertical mesh line not contained in $\mathcal{A}^{N}$.

We see that the slope of the gradient in the region $I I$ near $\partial \mathcal{A}^{N}$ is roughly $(2-$ $1.969) / h=0.031 \times 32=0.992$ which is evidence for the conjecture that $|\nabla u|=1$ along the straight segments of $\partial \mathcal{A}$. In fact, as remarked on page 75 of Buttazzo et al (1995), or 


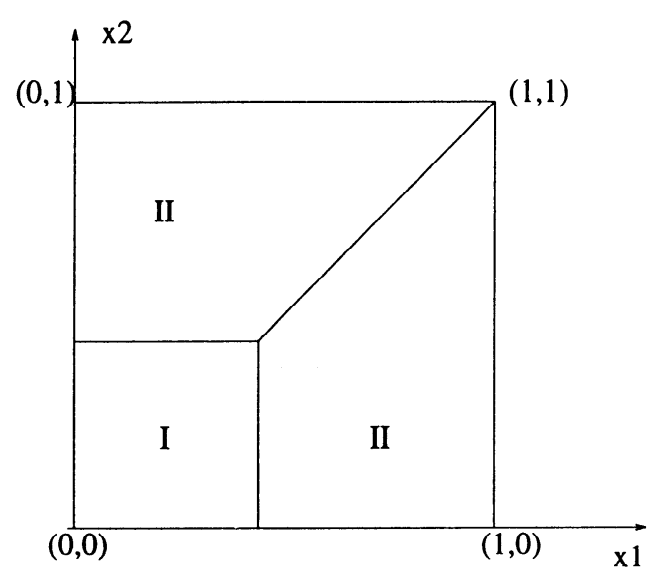

FIG. 5. Scheme of the subdomains $I$ and $I I$.

TABLE 2

Nodal coordinates $\left(u_{\varepsilon}^{N}\right)_{i j}$ for $M=2$ and $j=9$.

\begin{tabular}{cccccccccc}
\hline$i$ & 0 & 4 & 8 & 12 & 16 & 20 & 24 & 28 & 32 \\
\hline$\left(u_{\varepsilon}^{N}\right)_{i, 9}$ & 1.969 & 1.969 & 1.969 & 1.84 & 1.69 & 1.52 & 1.33 & 1.10 & 0.77 \\
\hline
\end{tabular}

TABLE 3

Numerical values for the relative resistance $R\left(u_{\varepsilon}^{N}\right) /|\Omega|$.

\begin{tabular}{cccc}
\hline$M$ & 1 & 2 & 3 \\
\hline$R\left(u_{\varepsilon}^{N}\right) /|\Omega|$ & 0.372 & 0.172 & .086 \\
\hline
\end{tabular}

page 478 of Whiteside (1974), Newton already knew that an optimal (rotational) body will have a blunt front end $\mathcal{A}$ with the slope of $u$ jumping from zero to one on $\partial \mathcal{A}$. Nobody seems to know, though, how Newton arrived at this insight, see Whiteside (1974). For nonrotational bodies (or nonradial minimizers $u$ ) it is natural to conjecture the same behaviour. This was discussed and partly proved in Buttazzo et al (1995), p 87.

We close with a listing of the numerical values for the relative resistance, defined by $R\left(u_{\varepsilon}^{N}\right) /|\Omega|$, for $\Omega=(-1,1)^{2}$ (see Table 3). 


\section{Concluding remarks}

The restriction to plane polygons $\Omega$ is not essential for the convergence result Theorem 2.1. If $\Omega$ is a convex polyhedron in $\mathbb{R}^{n}, n>2$, the proof of Theorem 2.1 still applies verbatim.

Although we considered explicitly only the case of polygonal domains and $f(x, u, p)=$ $f(p)$ in the present paper, our proofs can be modified in the case of curvilinear boundaries and general densities $f$, provided $f$ is a sufficiently smooth function of its arguments. The convergence analysis of higher-order elements for (1.6) can be based on the ideas in the present paper, but several new technical issues, such as numerical integration and proper enforcement of the constraint $0 \leqslant u(x) \leqslant M$ must still be resolved.

\section{Acknowledgements}

The research of BK was supported in part by the German Research Foundation (DFG) via the 'Sonderforschungsbereich No 359: Reaktive Strömungen, Diffusion \& Transport'; that of CS by IBM Germany with the resources of the IBM Scientific Centre, Heidelberg, where part of the paper was written while CS was a visiting researcher there. CS also gratefully acknowledges partial support from the AFOSR under grant No F49620-J-0100.

\section{REFERENCES}

ADAMS, R. A. 1978 Sobolev Spaces. New York: Academic.

BERTSEKAS, D. 1982 Projected Newton methods for optimization problems with simple constraints. SIAM J. Control Optimiz. 20, 221-246.

ButTAzzo, G., FERONE, V., \& KAwOHL, B. 1995 Minimum problems over sets of concave functions and related questions. Math. Nachrichten 173, 71-89.

CIARLET, P. G. 1978 The Finite Element Method for Elliptic Problems. Amsterdam: North-Holland.

DACOROGNA, B. 1989 Direct Methods in the Calculus of Variations (Springer Applied Mathematical Sciences 78). Berlin: Springer.

FEISTAUER, M., \& NEČAS, J. 1985 On the solvability of transonic potential flow problems. $Z$. Anal. Anwendungen 4, 305-329.

LiONS, P. L. 1981 Generalized Solutions of Hamilton-Jacobi Equations (Research Notes in Mathematics 69). Boston: Pitman.

MARCELLINI, P. 1990 Nonconvex integrals in the calculus of variations. Proc. of "Methods of Nonconvex Analysis" (Varenna, 1989) (Springer Lecture Notes in Mathematics 1440) Heidelberg: Springer (A. Cellina, ed). pp 16-57.

Miele, A. 1965 Theory of Optimum Aerodynamic Shapes. New York: Academic.

Newton, I. 1686 Philosophiae Naturalis Principia Mathematica.

RoCKAfEllaR, R. T. 1970 Convex Analysis (Princeton Mathematics Series vol. 28). Princeton, NJ: Princeton University Press.

Whiteside, D. T. 1974 The Mathematical Papers of Isaac Newton vol. VI. London: Cambridge University Press. 